\title{
Expression of PPAR- $\boldsymbol{\gamma}$ in adipose tissue of rats with polycystic ovary syndrome induced by DHEA
}

\author{
YU-XIA WANG ${ }^{1}$, WEI-JIE ZHU ${ }^{2}$ and BAO-GUO XIE ${ }^{2}$ \\ ${ }^{1}$ Department of Obstetrics and Gynecology, The First Affiliated Hospital, Jinan University, Guangzhou, \\ Guangdong 510630; ${ }^{2}$ Department of Developmental and Regenerative Biology, College of Life \\ Science and Technology, Jinan University, Guangzhou, Guangdong 510632, P.R. China
}

Received October 13, 2013; Accepted December 17, 2013

DOI: $10.3892 / \mathrm{mmr} .2014 .1895$

\begin{abstract}
The objective of this study was to investigate the expression of peroxisome proliferator-activated receptor- $\gamma$ (PPAR- $\gamma$ ) in adipose tissue of the rat model of polycystic ovary syndrome (PCOS), induced by dehydroepiandrosterone (DHEA). Sixteen sexually immature Sprague-Dawley female rats were randomly assigned to the DHEA $(n=8)$ or control $(n=8)$ group. Adipose tissue was collected from the two rat groups following subcutaneous injection of DHEA in the DHEA group and a standard laboratory diet in the control group for 20 consecutive days. Reverse transcription polymerase chain reaction and western blot analysis were performed to detect expression of PPAR- $\gamma$ at the mRNA and protein level in the adipose tissue. Both PPAR- $\gamma$ mRNA and protein levels were decreased in the adipose tissue of DHEA-induced PCOS rats compared to the control group. This decrease was significant $(\mathrm{P}<0.01)$. These results suggest that the pathogenesis of PCOS, which shares a number of common features with hyperandrogenemia, may involve the lipid metabolism pathway through inhibition of PPAR- $\gamma$.
\end{abstract}

\section{Introduction}

Peroxisome proliferator-activated receptor- $\gamma$ (PPAR- $\gamma$ ) is an essential nuclear receptor that acts as a key regulator of energy balance and is associated with hyperandrogenemia $(1,2)$. Moreover, the PPAR- $\gamma$ protein plays a pivotal role in modulating adipocyte differentiation, glucose and lipid homeostasis. Activation of PPAR $-\gamma$ improves the action of insulin and reduces the risk of obesity by modulating adipocyte differentiation, glucose and lipid homeostasis $(3,4)$. Deregulation of PPAR $-\gamma$ was associated with metabolic diseases, including

Correspondence to: Professor Wei-Jie Zhu, Department of Developmental and Regenerative Biology, College of Life Science and Technology, Jinan University, Room 839, Guangzhou, Guangdong 510632, P.R. China

E-mail: tzhuwj@jnu.edu.cn

Key words: peroxisome proliferator-activated receptor- $\gamma$, adipocytes, dehydroepiandrosterone, polycystic ovary syndrome obesity, type 2 diabetes, and obesity-associated hypertension (5).

Polycystic ovary syndrome (PCOS) is a common endocrine disorder in women of fertile age. PCOS is characterized by menstrual irregularity, ovarian and adrenal androgen overproduction, and insulin resistance (IR) (6). IR was recently recognized as a key etiological factor in metabolic disorders such as obesity, type 2 diabetes, and obesity-associated hypertension. A previous study indicated that gene polymorphisms in the PPAR- $\gamma$ gene are associated with PCOS occurrence in different ethnic backgrounds (7). The PPAR- $\gamma$ gene is mainly expressed in the adipose tissue, where it promotes the differentiation of preadipocytes into adipocytes and affects insulin sensitivity (8). A study exploring the correlation between the PPAR- $\gamma$ level and hyperinsulinemia suggested that hyperandrogenemia might be involved in the development of PCOS (9). The study showed that PPAR- $\gamma$ associates with PCOS pathogenesis. However, the expression of PPAR- $\gamma$ in adipose tissue of women with PCOS has not been fully understood.

The level of the androgen dehydroepiandrosterone (DHEA) is high in the blood of women with PCOS, and thus, DHEA is applied to establish animal models of PCOS (10). The DHEA-PCOS murine model exhibits many of the salient features of human PCOS such as hyperandrogenemia, IR, endocrine disturbance, follicle maturation disorders, and infertility (11). Moreover, the DHEA-PCOS model has been widely used to study a number of adipocytokines from adipose tissue, such as adiponectin, resistin, leptin and tumor necrosis factor- $\alpha$ (TNF- $\alpha$ ), which may influence the pathogenesis of IR in the PCOS (12).

The present study was designed to investigate PPAR- $\gamma$ expression in adipose tissue, and whether PPAR- $\gamma$ induces or attenuates, through the lipid metabolism pathway, the PCOS stimulated by DHEA. Evaluating this aspect would allow understanding of the relationship between adipocyte differentiation and the development of PCOS, as well as provide information relevant to the improvement of the efficacy of treatment of PCOS in certain conditions.

\section{Materials and methods}

Animals. Sixteen sexually immature Sprague-Dawley female rats (21 days old) were purchased from the Experimental Animal 
Center of Guangdong Province (Guangzhou, China). The rats were kept in a light-controlled room under a $12 \mathrm{~h} / 12 \mathrm{~h}$ light/ dark cycle and controlled temperature $\left(23-25^{\circ} \mathrm{C}\right)$, and had free access to food and water. The animals were randomly divided into the DHEA $(n=8)$ or control $(n=8)$ group. The rats in the DHEA group were subcutaneously injected with $6 \mathrm{mg} / 100 \mathrm{~g}$ body weight DHEA (Hubei Fangtong Pharmaceutical Co., Ltd., Huangshi, Hubei, China) dissolved in $0.2 \mathrm{ml}$ of sesame oil. Injections were performed daily for 20 consecutive days according to the method of Henmi et al (13). The rats in the control group received a standard laboratory diet for 20 consecutive days. During the experiment, the 4-day ovarian cycle of rats was monitored daily using vaginal cytology. The protocol was approved by the Ethics Committee of the First Affiliated Hospital of Jinan University (Guangzhou, China).

Tissue collection. All rats were sacrificed by decapitation $24 \mathrm{~h}$ following administration of the last DHEA dose. Parametrial adipose tissue was rapidly excised and immediately frozen in liquid nitrogen or stored at $-70^{\circ} \mathrm{C}$ until further use for reverse transcription polymerase chain reaction (RT-PCR) and western blotting.

$R T$-PCR. Total mRNA from adipose tissue was extracted from 16 fresh adipose tissue samples from the DHEA group $(n=8)$ and the control group ( $n=8)$ using an RNA extraction kit (Omega Bioteh, Norcross, GA, USA) according to the manufacturer's instructions. The adipose tissue were pulverized with a pestle and mortar in liquid nitrogen. The mRNA concentration and purity were determined by a $1.0 \%$ agarose gel electrophoresis and spectrophotometric measurements; the optical density (OD) ratio at $260 / 280 \mathrm{~nm}$ was $>1.8$. Aliquots of mRNA $(20 \mu \mathrm{g})$ from each sample were reverse transcribed using Oligo(dT $)_{18}$ primer and Moloney murine leukemia virus (MMLV) reverse transcriptase. The gene encoding $\beta$-actin was used as an internal control to normalize the results for variation in RNA quantities or differencies in the efficiency of reverse transcription. The primers used for amplification were PPAR- $\gamma$, forward 5'-GGTGAAACTCTGGGAGATCCTCC-3' and reverse 5'-AGCAACCATTGGGTCAGCTCT-3'; $\beta$-actin, forward 5'-CCTAAGGCCAACCGTAAAG-3' and reverse 5'-GGTCCACATTCTTTTCCTGATACTG-3'. Forty cycles of amplification were performed and each cycle consisted of denaturation at $95^{\circ} \mathrm{C}$ for $30 \mathrm{sec}$, annealing at $60^{\circ} \mathrm{C}$ for $1 \mathrm{~min}$, and extension at $70^{\circ} \mathrm{C}$ for $1 \mathrm{~min}$, with an additional extension at $72^{\circ} \mathrm{C}$ for $10 \mathrm{~min}$. Five microliters of each RT-PCR product were loaded onto a $1.5 \%$ agarose gel, and subsequently visualized and quantified using GDS-8000 Gel Scientific Image System and ImageQuant analysis software (Amersham Pharmacia Biotech, Hong Kong, China). Densitometrical values were used to calculate the ratio of PPAR- $\gamma$ to $\beta$-actin.

Western blotting. The adipose tissue samples were lysed on ice using cell lysis buffer and a protease inhibitor cocktail. After centrifugation at $10,000 \mathrm{x}$ g for $20 \mathrm{~min}$ at $4^{\circ} \mathrm{C}$, protein concentrations were determined using the Pierce Bicinchoninic Acid Protein Assay kit by Thermo Scientific (Rockford, IL, USA). Total protein from each sample was denatured in loading buffer, fractionated on a 10\% 1-dimensional SDS-PAGE gel, and transferred to a polyvinylidene difluoride membrane
(Immobilon-P; Millipore Corp., Bedford, MA, USA). Blots were blocked for $2 \mathrm{~h}$ in TBST solution $(20 \mathrm{mmol} / 1 \mathrm{Tris} \mathrm{pH}$ 7.6, $137 \mathrm{mmol} / 1$ sodium chloride, $0.1 \%$ Tween-20) supplemented with $10 \%$ non-fat dry milk. The blots were then incubated with antibodies against human PPAR- $\gamma(1: 1,000$; Cell Signaling Technology, Danvers, MA, USA) overnight at $4^{\circ} \mathrm{C}$, and against $\beta$-actin (1:3,000; Santa Cruz Biotechnology, Inc., Santa Cruz, CA, USA) for $1 \mathrm{~h}$ at room temperature by agitating. The blots were washed three times for $7 \mathrm{~min}$ each in TBST, followed by incubation for $1 \mathrm{~h}$ at room temperature with anti-rabbit and anti-mouse IgG horseradish peroxidase-conjugated species-specific secondary antibodies. The bound antibodies were detected using the BeyoECL Plus enhanced chemiluminescence system (Beyotime, Shanghai, China). Band intensities were quantified by scanning densitometry using the Quantity One software (Bio-Rad Laboratories, Hercules, CA, USA).

Immunohistochemistry. Paraffin-embedded sections of adipose tissue from the DHEA group $(\mathrm{n}=8)$ and the control group $(n=8)$ were used for immunohistochemistry. Positive staining was evaluated with the standard streptavidin-biotin system (Maixin Bio, Fuzhou, China). Sections $(4-\mu \mathrm{m})$ were deparaffinized in xylene, hydrated through graded alcohol, and incubated in antigen retrieval solution $(0.01-\mathrm{mol} / 1$ sodium citrate buffer, $\mathrm{pH} 6.0$ ) at $60^{\circ} \mathrm{C}$ for $16 \mathrm{~min}$. Endogenous peroxidase activity was blocked by incubating the samples in 3\% hydrogen peroxide for $10 \mathrm{~min}$. Non-specific antibody binding was blocked by incubation in normal goat serum for $10 \mathrm{~min}$. The PPAR- $\gamma$ monoclonal antibody (1:100, Maixin Bio) was used as the primary antibody, and the sections were incubated with this antibody overnight at $4^{\circ} \mathrm{C}$. As negative control, we used sections of the same tissues incubated without the primary antibody. As secondary antibodies, biotinylated anti-mouse immunoglobulins were used, and the reaction was developed using the streptavidin-peroxidase system. The diaminobenzidine substrate-chromogen system (Maixin Bio, Fuzhou, China) was used as the color-developing substrate.

Statistical analysis. Data are expressed as mean \pm standard deviation (SD). The difference between the two groups was evaluated by a t-test. $\mathrm{P}<0.05$ was considered to indicate statistical significance. Statistical analysis was performed using the SPSS 16.0 (SPSS, Chicago, IL, USA).

\section{Results}

RT-PCR analysis. PPAR- $\gamma$ gene expression in the adipose tissue was analyzed by RT-PCR. A total of 16 adipose tissue samples were positive for the $\beta$-actin mRNA, and thus considered for subsequent examination of the mRNA level of $P P A R-\gamma$ (Fig. 1). The expression level of PPAR- $\gamma$ in the DHEA group $(12.83 \pm 3.87)$ was significantly $(\mathrm{P}<0.01)$ lower $(28.83 \pm 4.15)$ compared to the control (Fig. 2).

Western blot analysis. PPAR- $\gamma$ protein expression in adipose tissue was analyzed by western blotting, and the level of the protein was normalized to that of $\beta$-actin (Fig. 3A). The result of quantitative analysis of the 16 adipose tissue samples is shown in Fig. 3B. The relative optical densities of the PPAR- $\gamma$ protein in the DHEA and the control group were $0.42 \pm 0.28$ 


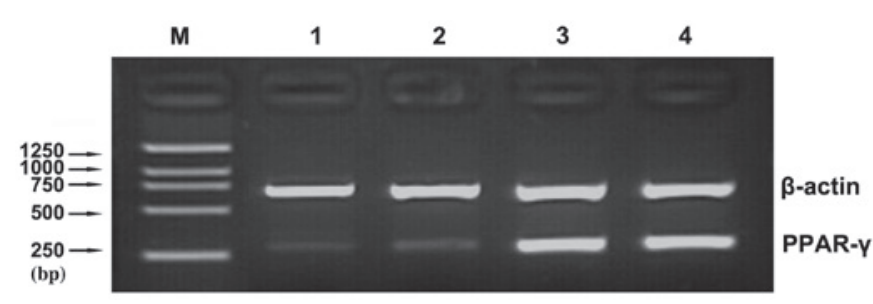

Figure 1. RT-PCR analysis of PPAR- $\gamma$ and $\beta$-actin mRNA levels in rat adipose tissue samples. PPAR $\gamma$ mRNA levels of adipose tissues were decreased in DHEA group. Representative intensities are shown in DHEA induced rats $(1,2)$ and control rats $(3,4) . \mathrm{M}$, marker; PPAR- $\gamma$, peroxisome proliferatoractivated receptor- $\gamma$.

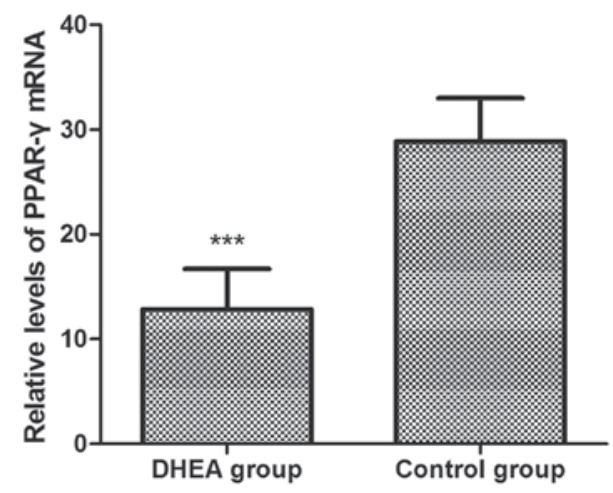

Figure 2. Relative quantities of $P P A R-\gamma$ mRNA levels in rat adipose tissues as measured by RT-PCR. The levels of PPAR- $\gamma$ mRNA were normalized to corresponding levels of $\beta$-actin, and values are shown as mean $\pm \mathrm{SD}$. $P P A R-\gamma$ mRNA levels were significantly decreased in adipose tissue of the DHEA group compared to the control group $\left({ }^{* * *} \mathrm{P}<0.01\right)$. PPAR $-\gamma$, peroxisome proliferator-activated receptor- $\gamma$; DHEA, dehydroepiandrosterone.

and $0.83 \pm 0.36$, respectively (Fig. 3B). The expression level of PPAR- $\gamma$ was thus lower in the DHEA group, and this decrease was significant $(\mathrm{P}<0.01)$.

Immunostaining of PPAR- $\gamma$ protein. PPAR- $\gamma$-positive immunostaining appeared in the adipose tissue of the two groups (Fig. 4). PPAR- $\gamma$ staining mainly appeared in the cytoplasm near the membrane of adipose cells. Positive staining of PPAR- $\gamma$ was also observed in the nuclei of adipose cells. PPAR- $\gamma$-positive immunostaining was strong in the control group and weak in the DHEA group.

\section{Discussion}

DHEA was found to be an abundant circulating androgen in women with PCOS (10). A mechanism underlying DHEA-induced PCOS involves endocrine disorder, which was related to abnormal levels of sex hormones (14). In previous studies, we successfully induced PCOS in rats using DHEA $(15,16)$. The nuclear hormone receptor PPAR- $\gamma$ has been widely used to elucidate the potential metabolic mechanisms underlying the induction of PCOS by DHEA. In the present study, PPAR- $\gamma$ expression was significantly lower in the DHEA group compared to the control group. This finding indicates that downregulation of PPAR- $\gamma$, induced by DHEA, might affect the pathogenesis of PCOS via yet undefined mechanisms.
A

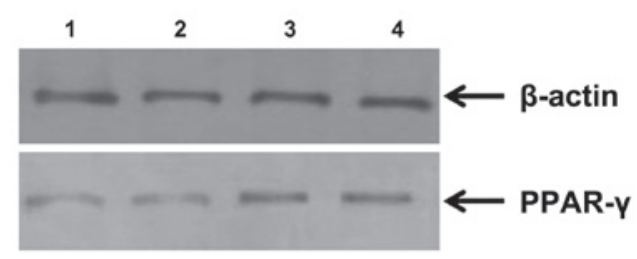

$\mathbf{B}$

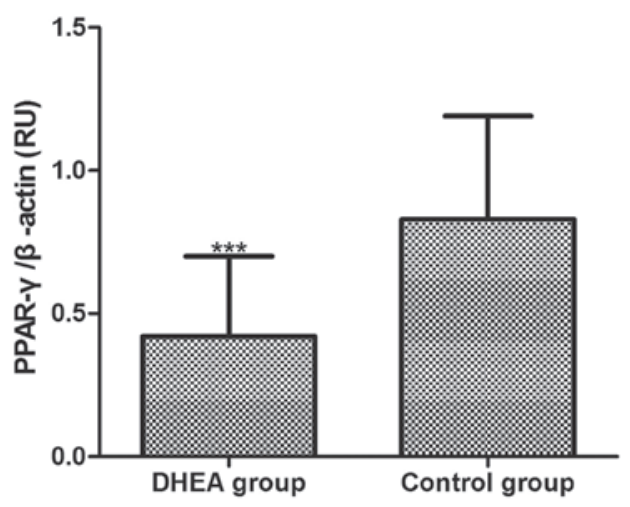

Figure 3. Western blot analysis of the PPAR- $\gamma$ and $\beta$-actin proteins (A) $\beta$-actin and PPAR- $\gamma$ protein were detected as the first and the second band on the blot, respectively. Representative intensities of PPAR- $\gamma$ protein are shown in DHEA-treated rats (lanes 1 and 2) and control rats (lanes 3 and 4). (B) Relative protein expression values were expressed as mean \pm SD. PPAR $-\gamma$ protein levels were significantly decreased in the DHEA group compared to the control group $\left({ }^{* * *} \mathrm{P}<0.01\right)$. PPAR- $\gamma$, peroxisome proliferator-activated receptor- $\gamma$; DHEA, dehydroepiandrosterone; RU, relative units.

The PPAR- $\gamma$ gene is suspected to be involved in the regulation of adipose metabolism in humans, and is also a susceptibility gene for the development of both obesity and IR, frequently associated with PCOS (17). Association of polymorphisms in the PPAR- $\gamma$ gene with PCOS in women remains a controversial issue, with significance of correlations depending on the population $(18,19)$. Therefore, whether there is a correlation between the expression of PPAR- $\gamma$ and PCOS, and whether there is another pathway via which the PPAR- $\gamma$ protein may exert its physiological effects on PCOS pathogenesis, independently of gene mutation or variation, is uncertain. We have demonstrated that expression of PPAR- $\gamma$ was significantly decreased in rats with PCOS induced by DHEA. The results of this study on the effect of PPAR- $\gamma$ in peripheral adipose tissue are consistent with the hypothesis that, apart from the direct endocrine function of androgen, hyperandrogenemia may induce metabolic abnormalities through regulation of PPAR- $\gamma$ in women with PCOS $(2,8)$. Moreover, previous studies have demonstrated that IR and hyperandrogenemia play a pathogenetic role in PCOS $(10,12,20)$. IR is a common feature of metabolic disorders including PCOS without exception of alterations in sensitivity to insulin which could be originated from PPAR- $\gamma(4,8,16)$.

Based on this study and the literature $(8,16,20,21)$, we present a model for the potential association between DHEA, PPAR- $\gamma$ and IR during the development of PCOS (Fig. 5). If some endogenous factors (e.g., hormone disturbance) or exogenous factors (e.g., environmental contamination by androgens and estrogens) raise the androgen level to a certain degree, the endocrine balance may be altered. Androgens, as DHEA in 

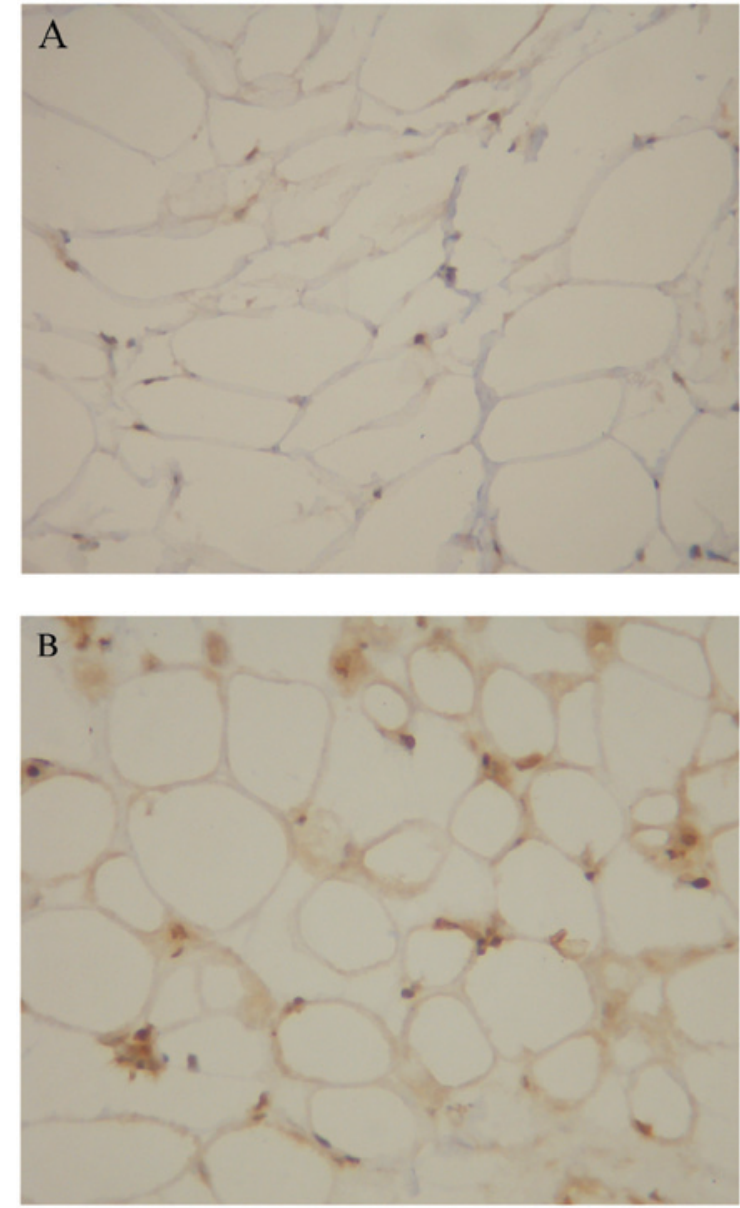

Figure 4. PPAR- $\gamma$ protein immunostaining in the adipose tissue. PPAR- $\gamma$ was detected in the cytoplasm of adipose tissue. Representative images from (A) the DHEA and (B) the control group are shown (magnification, $\mathrm{x} 200$ ). PPAR- $\gamma$, peroxisome proliferator-activated receptor- $\gamma$; DHEA, dehydroepiandrosterone.

this study, may directly affect the action of insulin by inhibiting the activity and expression of glucose-6-phosphatase and phosphoenolpyruvate carboxykinase (PEPCK). DHEA increases glucose uptake in hepatocytes and increases insulin binding to its own receptor leading to hyperinsulinism, and eventually, IR (22). It is generally accepted that IR is likely to aggravate clinical features of PCOS. On the other hand, DHEA may contribute to PCOS via another pathway, through reduction of the expression levels of PPAR- $\gamma$. In vivo and in vitro studies have demonstrated that DHEA activates phosphatidylinositol 3-kinase and atypical protein kinase $\mathrm{C}$ (PKC) via $\mathrm{C} / \mathrm{EBP} \alpha$ transcription factors to decrease the expression of PPAR- $\gamma$. The reduction in PPAR- $\gamma$ protein levels might attenuate other adipocyte-specific phenotypes through changes in the spatial conformation of proteins such as glyceraldehyde 3-phosphate dehydrogenase (GAPDH), adipocyte lipidbinding protein $(\mathrm{aP} 2)$ and sterol regulatory element binding protein (SREBP) $(23,24)$. These proteins are transcriptional factors associated with hyperinsulinism, inevitably promoting PCOS. In addition, IR in PCOS reduces the concentration of peripheral tissues in PPAR- $\gamma$ via TNF- $\alpha$, which is another PPAR- $\gamma$-reducing agonist (25). The subsequent decrease in PPAR- $\gamma$ expression further initiates the occurrence of a number of IR symptoms. Thus, we suggest that a complex

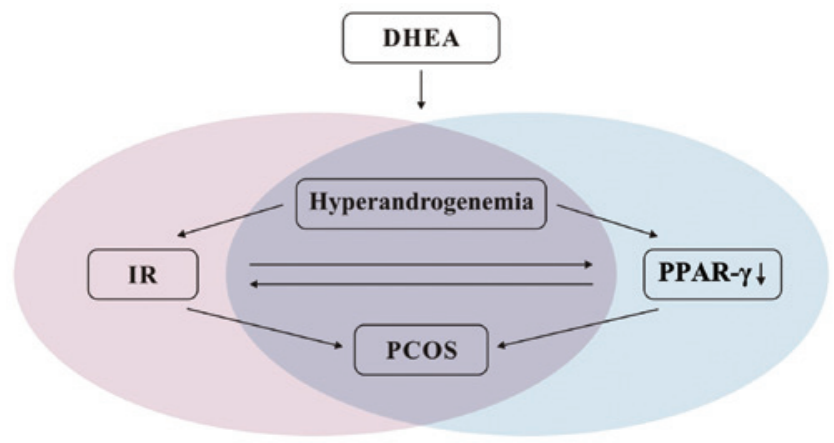

Figure 5. Model for the association between DHEA, PPAR- $\gamma$ and IR in PCOS Exogenous DHEA increases the androgen level. On the one hand, hyperandrogenemia leads to IR, which can aggravate clinical features of PCOS. On the other hand, hyperandrogenemia can decrease the expression of PPAR- $\gamma$ in adipose tissue via the lipid metabolism pathway, further contributing to PCOS pathogenesis. The reduction in PPAR- $\gamma$ can further result in hyperinsulinism, initiating the occurrence of several symptoms of IR, and IR in PCOS may decrease the PPAR- $\gamma$ level. Therefore, multiple interactions and two pathways are associated with the occurrence or development of PCOS under conditions of excess of DHEA. DHEA, dehydroepiandrosterone; IR, insulin resistance; PCOS, polycystic ovary syndrome; PPAR- $\gamma$, peroxisome proliferator-activated receptor- $\gamma$.

and multiple network of interactions may characterize and regulate PPAR- $\gamma$, hyperandrogenemia, IR and PCOS. As shown in Fig. 5, hyperandrogenemia induces PCOS through the classical IR pathway. Based on our results, the occurrence or development of PCOS in conditions of androgen excess may also correlate to the lipid metabolism pathway associated with the decrease in PPAR- $\gamma$. There is number of limitations in the present study, such as the limited sample size and the fact that we used an animal model. Therefore, whether additional pathways or negative feedback loops allow to bypass the regulation scheme presented in Fig. 5 remains to be investigated.

Expression patterns of $P P A R-\gamma$ differ substantially in numerous diseases and tissues. In rat models of diabetes, the PPAR- $\gamma$ gene was downregulated in the renal cortex and retina (26), while it was upregulated in the aorta (27). By contrast, in the present study, only a reduction in the transcription and translation of the gene was observed in DHEA-induced rats with PCOS, with concordant results from three independent methods of assessment of the expression of PPAR- $\gamma$. This indicates that PPAR- $\gamma$ may exert various biological effects dependent on the tissue and other biological features. Thus, to understand the exact physiological effects of PPAR- $\gamma$ on infertility of PCOS women, the variability and complexity of PPAR- $\gamma$ expression need to be thoroughly studied. Although PPAR- $\gamma$ is primarily expressed in adipose tissue, it can have direct or indirect effects on regulation of the function of ovarian granulosa cells, by affecting related adipocytokines that affect the normal release of oocytes (28). Moreover, because of the alteration in lipid metabolism caused by impaired insulin signaling, regulation, by PPAR- $\gamma$, of its downstream targets is also impaired (29). These two effects induced by PPAR- $\gamma$ on ovary and lipid metabolism may constitute important factors contributing to the infertility of women with PCOS. PPAR- $\gamma$ may thus play a role in the molecular linking of lipid metabolism to reproduction, with inactivation of PPAR- $\gamma$ promoting infertility in women with PCOS. Thus, treating PCOS requires focalizing on, and understanding the 
PPAR- $\gamma$-related pathways, and potential therapeutic agents targeting this protein to enhance its activity have the potential to improve fertility.

In summary, results of this study have shown that DHEA excess can induce an adipose-specific reduction in PPAR- $\gamma$ expression, which is associated with PCOS. The characteristics of PPAR- $\gamma$ expression in adipose tissue of the PCOS rat model indicated that PPAR $-\gamma$ may induce or promote PCOS through the lipid metabolism pathway. A complex network might promote development of PCOS, which in this study was associated with an increase in DHEA and a decrease in PPAR- $\gamma$ levels. Both the metabolic and endocrine pathways are related to the pathogenesis of PCOS. Further studies are needed to elucidate the etiology of infertility and its association with PPAR- $\gamma$ expression in PCOS. Improved therapeutic treatment in the clinic may involve developing agents specifically targeting PPAR- $\gamma$ to increase fertility of women with PCOS.

\section{Acknowledgements}

This study was supported by Science and Technology Planning Project of Guangdong Province, China (no. 2012B031800400).

\section{References}

1. Ryan KK, Li B, Grayson BE, Matter EK, Woods SC and Seeley RJ: A role for central nervous system PPAR- $\gamma$ in the regulation of energy balance. Nat Med 17: 623-626, 2011.

2. Cipolletta D, Feuerer M, Li A, Kamei N, Lee J, Shoelson SE, Benoist $C$ and Mathis D: PPAR- $\gamma$ is a major driver of the accumulation and phenotype of adipose tissue Treg cells. Nature 486 549-553, 2012.

3. Sun K and Scherer PE: The PPAR $\gamma$-FGF1 axis: an unexpected mediator of adipose tissue homeostasis. Cell Res 22: 1416-1418, 2012.

4. Jonker JW, Suh JM, Atkins AR, Ahmadian M, Li P, Whyte J, He M, Juguilon H, Yin YQ, Phillips CT, Yu RT, Olefsky JM, Henry RR, Downes M and Evans RM: A PPAR $\gamma$-FGF1 axis is required for adaptive adipose remodelling and metabolic homeostasis. Nature 485: 391-394, 2012.

5. Tyagi S, Gupta P, Saini AS, Kaushal C and Sharma S: The peroxisome proliferator-activated receptor: a family of nuclear receptors role in various diseases. J Adv Pharm Technol Res 2: 236-240, 2011.

6. Svendsen PF, Christiansen M, Hedley PL, Nilas L, Pedersen SB and Madsbad S: Adipose expression of adipocytokines in women with polycystic ovary syndrome. Fertil Steril 98: 235-241, 2012.

7. Dasgupta S, Sirisha P, Neelaveni K, Anuradha K, Sudhakar G and Reddy BM: Polymorphisms in the IRS- 1 and PPAR- $\gamma$ genes and their association with polycystic ovary syndrome among South Indian women. Gene 503: 140-146, 2012.

8. Saraf N, Sharma PK, Mondal SC, Garg VK and Singh AK: Role of PPARg2 transcription factor in thiazolidinedione-induced insulin sensitization. J Pharm Pharmacol 64: 161-171, 2012.

9. Amalfi S, Velez LM, Heber MF, Vighi S, Ferreira SR, Orozco AV, Pignataro $\mathrm{O}$ and Motta AB: Prenatal hyperandrogenization induces metabolic and endocrine alterations which depend on the levels of testosterone exposure. PLoS One 7: e37658, 2012.

10. Lenarcik A, Bidzińska-Speichert B, Tworowska-Bardzińska U and Krępuła K: Hormonal abnormalities in first-degree relatives of women with polycystic ovary syndrome (PCOS). Endokrynol Pol 62: 129-133, 2011.

11. Abramovich D, Irusta G, Bas D, Cataldi NI, Parborell F and Tesone M: Angiopoietins/TIE2 system and VEGF are involved in ovarian function in a DHEA rat model of polycystic ovary syndrome. Endocrinology 153: 3446-3456, 2012.

12. Glintborg D, Andersen M, Hagen C, Frystyk J, Hulstrøm V, Flyvbjerg A and Hermann AP: Evaluation of metabolic risk markers in polycystic ovary syndrome (PCOS). Adiponectin, ghrelin, leptin and body composition in hirsute PCOS patients and controls. Eur J Endocrinol 155: 337-345, 2006.
13. Henmi H, Endo T, Nagasawa K, Hayashi T, Chida M, Akutagawa N, Iwasaki M, Kitajima Y, Kiya T, Nishikawa A, Manase K and Kudo R: Lysyl oxidase and MMP-2 expression in dehydroepiandrosterone-induced polycystic ovary in rats. Biol Reprod 64: 157-162, 2001.

14. Rosenfield RL, Mortensen M, Wroblewski K, Littlejohn E and Ehrmann DA: Determination of the source of androgen excess in functionally atypical polycystic ovary syndrome by a short dexamethasone androgen-suppression test and a low-dose ACTH test. Hum Reprod 26: 3138-3146, 2011.

15. Wang YX, Xie XM and Zhu WJ: Serum adiponectin and resistin levels in patients with polycystic ovarian syndrome and their clinical implications. J Huazhong Univ Sci Technolog Med Sci 30: 638-642, 2010.

16. Wang YX, Sun YY and Qiu HY: Expression of resistin mRNA in adipose tissue of rat model with polycystic ovarian syndrome and its implication. J Huazhong Univ Sci Technolog Med Sci 24: 621-624, 2004

17. Knebel B, Lehr S, Janssen OE, Hahn S, Nitzgen U, Jacob S, Haas J, Muller-Wieland D and Kotzka J: Genetic variants in central metabolic genes influence some but not all relations of inflammatory markers in a collective with polycystic ovary syndrome. Arch Physiol Biochem 118: 219-229, 2012.

18. Zhang H, Bi Y, Hu C, Lu W and Zhu D: Association between the Pro12Ala polymorphism of PPAR- $\gamma$ gene and the polycystic ovary syndrome: a meta-analysis of case-control studies. Gene 503: 12-17, 2012.

19. San-Millán JL and Escobar-Morreale HF: The role of genetic variation in peroxisome proliferator-activated receptors in the polycystic ovary syndrome (PCOS): an original case-control study followed by systematic review and meta-analysis of existing evidence. Clin Endocrinol (Oxf) 72: 383-392, 2010.

20. Brettenthaler N, De Geyter C, Huber PR and Keller U: Effect of the insulin sensitizer pioglitazone on insulin resistance, hyperandrogenism, and ovulatory dysfunction in women with polycystic ovary syndrome. J Clin Endocrinol Metab 89: 3835-3840, 2004.

21. Yildiz BO, Yarali H, Oguz H and Bayraktar M: Glucose intolerance, insulin resistance, and hyperandrogenemia in first degree relatives of women with polycystic ovary syndrome. J Clin Endocrinol Metab 88: 2031-2036, 2003.

22. Brennan K, Huang A and Azziz R: Dehydroepiandrosterone sulfate and insulin resistance in patients with polycystic ovary syndrome. Fertil Steril 91: 1848-1852, 2009.

23. Kajita K, Ishizuka T, Mune T, Miura A, Ishizawa M, Kanoh Y, Kawai Y, Natsume Y and Yasuda K: Dehydroepiandrosterone down-regulates the expression of peroxisome proliferatoractivated receptor gamma in adipocytes. Endocrinology 144: 253-259, 2003.

24. Yogosawa S, Mizutani S, Ogawa Y and Izumi T: Activin receptorlike kinase 7 suppresses lipolysis to accumulate fat in obesity through downregulation of peroxisome proliferator-activated receptor $\gamma$ and C/EBP $\alpha$. Diabetes 62: 115-123, 2012.

25. Swaroop JJ, Rajarajeswari D and Naidu JN: Association of TNF- $\alpha$ with insulin resistance in type 2 diabetes mellitus. Indian J Med Res 135: 127-130, 2012.

26. Wang F, Gao L, Gong B, Hu J, Li M, Guan Q and Zhao J: Tissue-specific expression of PPAR mRNAs in diabetic rats and divergent effects of cilostazol. Can J Physiol Pharmacol 86: 465-471, 2008.

27. Sharma AK, Bharti S, Ojha S, Bhatia J, Kumar N, Ray R, Kumari S and Arya DS: Up-regulation of PPAR $\gamma$, heat shock protein-27 and -72 by naringin attenuates insulin resistance, $\beta$-cell dysfunction, hepatic steatosis and kidney damage in a rat model of type 2 diabetes. Br J Nutr 106: 1713-1723, 2011.

28. Kim J, Sato M, Li Q, Lydon JP, Demayo FJ, Bagchi IC and Bagchi MK: Peroxisome proliferator-activated receptor gamma is a target of progesterone regulation in the preovulatory follicles and controls ovulation in mice. Mol Cell Biol 28: 1770-1782, 2008.

29. Asrih M, Lerch R, Papageorgiou I, Pellieux C and Montessuit C: Differential regulation of stimulated glucose transport by free fatty acids and PPAR $\alpha$ or $-\delta$ agonists in cardiac myocytes. Am J Physiol Endocrinol Metab 302: E872-E884, 2012. 